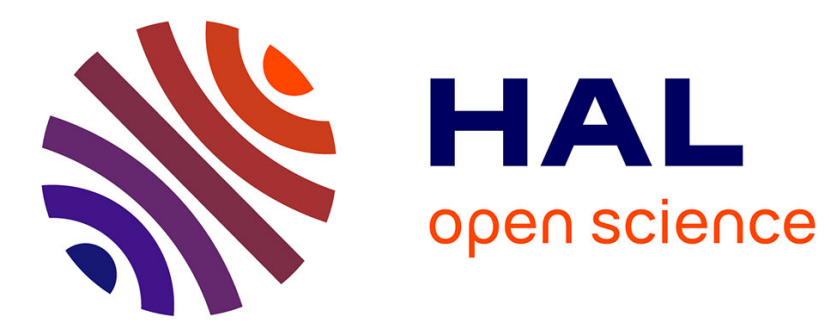

\title{
La photographie de presse au ban des dispositifs d'exposition numérique. Paris Match et l'AFP sur Instagram
}

\author{
Maxime Fabre
}

\section{- To cite this version:}

Maxime Fabre. La photographie de presse au ban des dispositifs d'exposition numérique. Paris Match et l'AFP sur Instagram. Communication \& langages, 2017. hal-02432658

\section{HAL Id: hal-02432658 \\ https://hal.science/hal-02432658}

Submitted on 8 Jan 2020

HAL is a multi-disciplinary open access archive for the deposit and dissemination of scientific research documents, whether they are published or not. The documents may come from teaching and research institutions in France or abroad, or from public or private research centers.
L'archive ouverte pluridisciplinaire HAL, est destinée au dépôt et à la diffusion de documents scientifiques de niveau recherche, publiés ou non, émanant des établissements d'enseignement et de recherche français ou étrangers, des laboratoires publics ou privés. 


\title{
LA PHOTOGRAPHIE DE PRESSE AU BAN DES DISPOSITIFS D'EXPOSITION NUMÉRIQUE. PARIS MATCH ET L'AFP SUR INSTAGRAM
}

\author{
Maxime Fabre
}

NecPlus | « Communication \& langages »

2017/4 N 194 | pages 87 à 103

ISSN 0336-1500

ISBN 9782358761840

Article disponible en ligne à l'adresse :

https://www.cairn.info/revue-communication-et-langages1-2017-4-page-87.htm

Distribution électronique Cairn.info pour NecPlus.

(C) NecPlus. Tous droits réservés pour tous pays.

La reproduction ou représentation de cet article, notamment par photocopie, n'est autorisée que dans les limites des conditions générales d'utilisation du site ou, le cas échéant, des conditions générales de la licence souscrite par votre établissement. Toute autre reproduction ou représentation, en tout ou partie, sous quelque forme et de quelque manière que ce soit, est interdite sauf accord préalable et écrit de l'éditeur, en dehors des cas prévus par la législation en vigueur en France. Il est précisé que son stockage dans une base de données est également interdit. 
La photographie de presse au ban des dispositifs d'exposition numérique. Paris Match et l'AFP sur

\section{Instagram}

\section{Photographie de presse et dispositifs nUméridues}

Depuis 1989 a lieu le festival international de photojournalisme Visa pour l'image à Perpignan. Chaque année, le festival organise des expositions, des projections et élabore des tables rondes où les professionnels de l'image discutent des enjeux qui animent et parfois bouleversent leur métier. En 2012, dans un entretien pour le British Journal of Photography, le directeur du festival JeanFrançois Leroy expose ses critiques vis-à-vis des nouveaux outils numériques, et notamment d'Instagram, application de partage d'images sur Internet :

Cette année, c'est la mode Hipstamatic, Instagram qui m'exaspère. Si je photographie une poubelle avec Instagram, la photo est jolie mais je n'y suis pour rien, c'est l'appareil qui fait tout. Où est l'œil du photographe? En revanche, quand Karim Ben Khelifa photographie en Syrie avec un iPhone, je comprends que c'est pour des raisons de sécurité. Mais on peut faire des photos avec un iPhone sans utiliser Instagram ou Hipstamatic. [...] Quel que soit l'outil, ce qui nous intéresse c'est l'œil. Hipstamatic, Instagram, c'est une paresse intellectuelle et ça devient un «truc ». Et ce n'est pas parce qu'on fait des photos à l'Hipstamatic que c'est un sujet ${ }^{1}$.

En 2014, c'est la dimension économique de ces nouveaux outils qui interroge les professionnels du métier, comme le rappelle la journaliste Patricia Blettery

\section{MAXIME FABRE}

La présente publication interroge le statut de la photographie de presse numérique à travers l'analyse des comptes Instagram de I'Agence France-Presse et de Paris Match. En proposant une application communicationnelle du terme "banal ", l'auteur suggère de comprendre la photographie de presse numérique comme l'enjeu d'une éco-sémiotique complexe, où dispositifs numériques et industries journalistiques établissent ensemble un espace de circulation et de passage du sens photographique.

Mots clés: sémiotique, communication, photojournalisme, dispositif, médias informatisés, Paris Match, AFP, banalité, Instagram

1. URL: http://www.2ebureau.com/03_culture/visa_pour_image/media/Communiques/Conversation_ JFL_2012.pdf 
pour Radio France International dans un article intitulé «Photographie et nouveaux médias : Instagram inquiète Visa pour l'image ${ }^{2} »$. En 2016 pourtant, le changement est brutal. Le festival dispose à présent d'un compte Instagram, quand le photojournaliste Andrew Quilty annonce qu'il a été contacté par le même Jean-François Leroy grâce au service de messagerie de l'application :

Théoriquement, j'ai 150000 personnes qui voient mes photos sur Instagram. Quel est le nombre de lecteurs d'un quotidien parisien ? Peut-être plus, mais c'est déjà énorme. C'est plus simple pour les directeurs photos de suivre mon travail. C'est comme ça que Jean-François Leroy m'a trouvé. C'est grâce à Instagram si je suis à Visa pour l'image ! ${ }^{3}$

À présent, les photojournalistes n'hésitent pas à rappeler qu'Instagram permet une visibilité accrue de leur travail ${ }^{4}$ auprès d'éventuels clients et d'un public de plus en plus important ${ }^{5}$. Si les photographes indépendants sont les premiers à s'emparer du dispositif, c'est ensuite au tour des grandes industries journalistiques de s'installer sur l'application. En 2014, l'Agence France-Presse et le magazine Paris Match se lancent mutuellement sur l'application à travers deux comptes distincts : @afpphoto pour la première ${ }^{6}$ et @ parismatch_magazine pour le second ${ }^{7}$.

Le rapport entre l'application et le photojournalisme est donc complexe. D'un côté les critiques se chargent de rappeler qu'Instagram est un outil de conditionnement esthétique des images et qu'il engage en parallèle un modèle de gratuité qui ne peut convenir à celui de la presse ; de l'autre les photojournalistes n'hésitent pas à l'utiliser dans un but de visibilité accrue de leur travail et de rapidité de transmission de leurs images, confirmant ainsi l'intérêt de l'application pour la profession.

Afin de comprendre ce qui se joue précisément dans la relation entre le dispositif numérique et la pratique photojournalistique, nous nous proposons de focaliser notre étude sur les comptes Instagram de Paris Match et de l'Agence France-Presse. L'objectif est d'étudier de quelle manière une application est capable de reconfigurer les codes éthiques et esthétiques du photojournalisme et inversement, comment l'investissement de ces deux industries journalistiques sur Instagram a conduit à ajuster - voire même renforcer - la présence de la photographie de presse sur le réseau social.

D’un point de vue théorique, il s'agira d'éprouver les relations sémiotiques et communicationnelles qui ont pu s'établir - et qui s'établissent encore par

2. URL : http://www.rfi.fr/culture/20140907-photographie-nouveaux-medias-instagram-visa-imagephotojournalisme-festival-perpignan-facebook-reseaux-sociaux

3. URL: http://esjmontpellier.com/blogs/visa2016/andrew-quilty-cest-grace-a-instagram-si-je-suis-avisa-pour-limage/

4. Le photographe David Guttenfelder pour Le Temps, lien : https://www.letemps.ch/culture/2016/ 09/01/instagram-change-vie-photographe

5. En 2016, Instagram revendique 700 millions d'abonnés à son application, lien : https://www.blogdumoderateur.com/chiffres-reseaux-sociaux/

6. URL : https://www.instagram.com/afpphoto/?hl=fr

7. URL : https://www.instagram.com/parismatch_magazine/?hl=fr 
contraste - entre un dispositif numérique d'un côté, et une agence de presse accompagnée d'un magazine d'actualité de l'autre.

En outre, l'analyse des comptes Instagram de Paris Match et de l'AFP nous permettra de comprendre ce qui se joue à travers les notions de partage et de départage dans la photographie de presse à l'épreuve des "réseaux sociaux de l'image ", que nous nous proposons de renommer "dispositifs d'exposition numérique », comme nous le verrons par la suite. L'enjeu n'est donc pas seulement sémiotique, il est aussi politique et économique ${ }^{8}$.

\section{LA QUESTION DU DISPOSITIF : LE (DÉS)-EMBOÎTEMENT DES MÉdIATIONS}

Instagram a été fondée en octobre de l'année 2010 par le développeur américain Kevin Systrom et l'ingénieur brésilien Mike Krieger. L'application est conçue pour les appareils mobiles du système d'exploitation iOS de la marque Apple, l'Android de Google et le Windows Phone de Microsoft ${ }^{9}$. En 2012, elle est rachetée par le réseau social en ligne Facebook au prix d'un milliard de dollars américains, alors que les observateurs estiment que ce montant est injustifié pour une application gratuite et sans revenus, comme en témoigne le journaliste Pierric Marrisal pour L'Humanité :

Lorsqu'une entreprise complètement surévaluée, Facebook, achète un milliard de dollars une entreprise qui ne génère aucun revenu, Instagram, on n'assiste pas à un miracle de l'Internet, mais bien à une folie spéculative ${ }^{10}$.

En décembre de la même année, pourtant, Facebook change les conditions d'utilisation d'Instagram en permettant ainsi la commercialisation des images exposées par les utilisateurs sur l'application. Les enjeux du rachat par Facebook sont alors plus clairs et mettent à présent en évidence les possibilités commerciales de l'application d'images. Si Facebook revient rapidement sur ces changements, suite aux mécontentements de ses utilisateurs, l'application a dévoilé son potentiel marchand ${ }^{11}$ et propose dorénavant des publicités officielles ${ }^{12}-$ comme $^{2}$ officieuses $^{13}$ - à ses utilisateurs.

D'un point de vue purement technique, Instagram n'a jamais cessé de faire évoluer les paramétrages de son application : la taille et la disposition des images sur l'application ont évolué du format carré au format paysage en 2015, les filtres proposés ont augmenté au fil des années - et cela dès 2011, la possibilité de filmer des événements en direct a été intégrée en 2016, la localisation des endroits précis

8. Voir Dominique Cardon, À quoi rêvent les algorithmes. Nos vies à l'heure des big data, Paris, Seuil, 2015.

9. L'application est aujourd'hui disponible sur Internet, sans toutefois en permettre une utilisation complète.

10. URL : http://www.humanite.fr/social-eco/regardons-une-bulle-speculative-gonfler-gonfler\%E2\% 80\%A6-494258

11. Voir Jason Miles, Instagram power : build your brand and reach more customers with the power of pictures, New-York, McGraw-Hill Education, 2013.

12. Les publicités sont présentées comme du contenu «sponsorisé ».

13. URL : https://www.letemps.ch/opinions/2017/04/25/publicite-deguisee-instagram-derange 


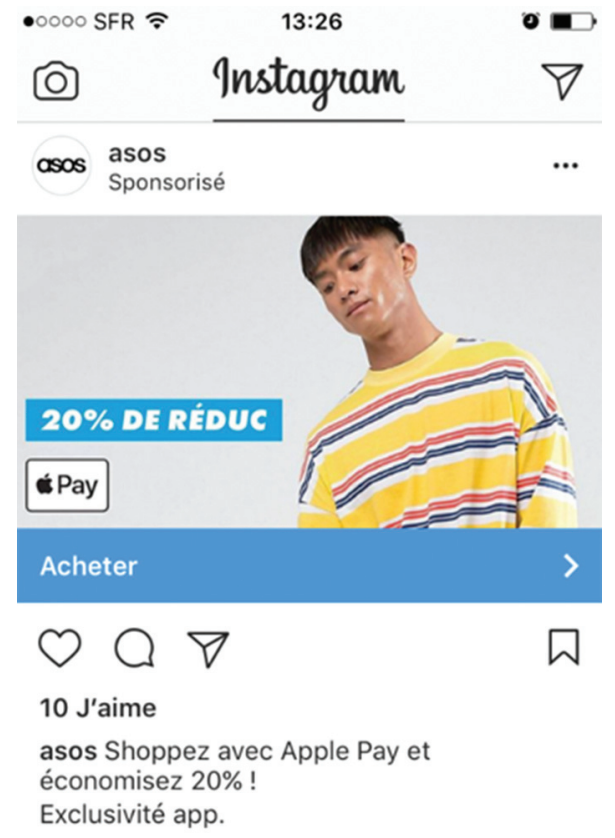

Figure 1 : La publicité « sponsorisée " sur Instagram, l'exemple de la marque de prêt-à-porter Asos ${ }^{14}$

de la planète dès 2012. Il est également possible de répertorier ses images à l'aide de hashtags dans la légende ou les commentaires liés à la photographie en $2013^{15}$.

Instagram est donc un dispositif d'exposition numérique. L'affirmation semble anodine, elle est pourtant cruciale, car de cette notion de " dispositif " découle un certain nombre de définitions, de caractères techno-sémiotiques et d'enjeux à la fois institutionnels, sociaux et marchands. Pour Michel Foucault, d'abord, un dispositif est « un ensemble résolument hétérogène comportant des discours, des institutions [...], des décisions réglementaires, des lois, des mesures administratives, des énoncés scientifiques ${ }^{16} »$. On vient de le voir, Instagram regroupe ces catégories. Mais la définition est encore trop descriptive pour devenir opérante dans notre analyse. Trente ans plus tard, Giorgio Agamben précise la visée de Michel Foucault en soulevant les enjeux sociotechniques du dispositif :

J'appelle dispositif tout ce qui a, d'une manière ou une autre, la capacité de capturer, d'orienter, de déterminer, d'intercepter, de modeler, de contrôler et d'assurer les gestes, les conduites, les opinions et les discours des êtres vivants ${ }^{17}$.

La définition d'Agamben nous permet d'envisager Instagram dans la relation qu'elle établit entre un objet et des " êtres vivants ». Car dans ce geste

14. Source : capture d'écran de l'application Instagram le 24 juillet 2017

15. URL : http://wersm.com/the-complete-history-of-instagram/

16. Michel Foucault, Dits et écrits, volume III, Paris, Gallimard, 1994, p. 299 et suivantes.

17. Giorgio Agamben, Qu'est-ce qu'un dispositif?, Paris, Rivages poche, 2014, quatrième de couverture. 
de « contrôle», le dispositif a un pouvoir sémiotique fort. Il « organise la communication ${ }^{18} »$ et la contraint en déterminant les pratiques de ses utilisateurs. Il est donc un objet à la fois technique, social, matériel et sémiotique.

Les enjeux de notre analyse sont à présent plus clairs. Pourtant, ces deux définitions restent encore très théoriques. C'est finalement l'approche polémologique des médias et de la consommation ${ }^{19}$ de Michel de Certeau qui nous permet de compléter un peu plus la notion de dispositif caractérisée plus haut, en rendant compte de la «poétique sociale ${ }^{20}$ » des pratiques numériques initiées par et sur Instagram. Dans notre cas, c'est l'opérativité du couple conceptuel stratégie/tactique définie dans L'invention du quotidien ${ }^{21}$ qui nous intéressera au cours de l'analyse, et qui autorise à penser le complexe techno-sémiotique qui se noue entre la détermination d'un dispositif d'un côté, et la "rhétorique des pratiques » de l'autre.

En résumé, la «stratégie » est un système de règles, d'éléments fixes et rigides énoncés par le dispositif, tandis que la tactique implique des éléments singuliers, un contexte, une pratique du temps et des occasions qui ne sont a priori pas pris en compte par la stratégie. La pratique journalistique de la photographie de presse sur Instagram en est un bon exemple, car elle met constamment en tension le couple stratégie/tactique en l'illustrant par l'interdépendance du dispositif et des pratiques qui s'y créent : entre la direction marketing imposée par l'application à la presse, et l'esthétique photojournalistique qui s'y développe en parallèle.

\section{Du PHOTOJOURNALISME TRAdITIONNEL À LA PHOTOGRAPHIE MOBILE}

La photographie de presse fait son entrée dans l'application entre 2012 et 2013, notamment grâce aux reportages de l'Américain Ben Lowy sur la guerre au Moyen-Orient et la couverture de l'ouragan Sandy par le Time. Mais ces pratiques restent minoritaires, car elles sont encore très critiquées par de nombreux photojournalistes, dont certains représentent des institutions importantes de la profession. Le 23 février 2012, le photographe Nick Stern publie un article au titre provocateur sur le site de CNN : "Why Instagram photos cheat the viewer ${ }^{22}$. Il y critique la pratique de Ben Lowy, notamment sur le caractère esthétique des photographies produites par l'intermédiaire d'un téléphone mobile, et leur utilisation des filtres - des manipulations de l'image qu'ils supposent rendue possible par l'application Instagram. Il n'est bien sûr pas le seul, dans son article intitulé « Hipstamatic and the Death of Photojournalism », Matt Buchanan critique une application similaire à celle d'Instagram, Hipstamatic, et l'utilisation qu'en fait le photographe de presse Damon Winter dans son reportage «A grunt's life » sur la vie des soldats américains en Afghanistan. Le débat est en fait celui de la légitimité professionnelle, comme l'explique Vincent Lavoie :

18. Yves Jeanneret, Critique de la trivialité, Le Havre, Éditions Non Standard, 2014, p. 11.

19. Point de vue qui affirme la conflictualité créative des pratiques culturelles dans l'opposition qu'il opère entre tactique et stratégie.

20. Ibid, p. 365.

21. Michel de Certeau, L'invention du quotidien. 1. arts de faire, Paris, Gallimard, p. 57-63.

22. URL : http://edition.cnn.com/2012/02/22/opinion/phones-instagram-apps-stern/ 

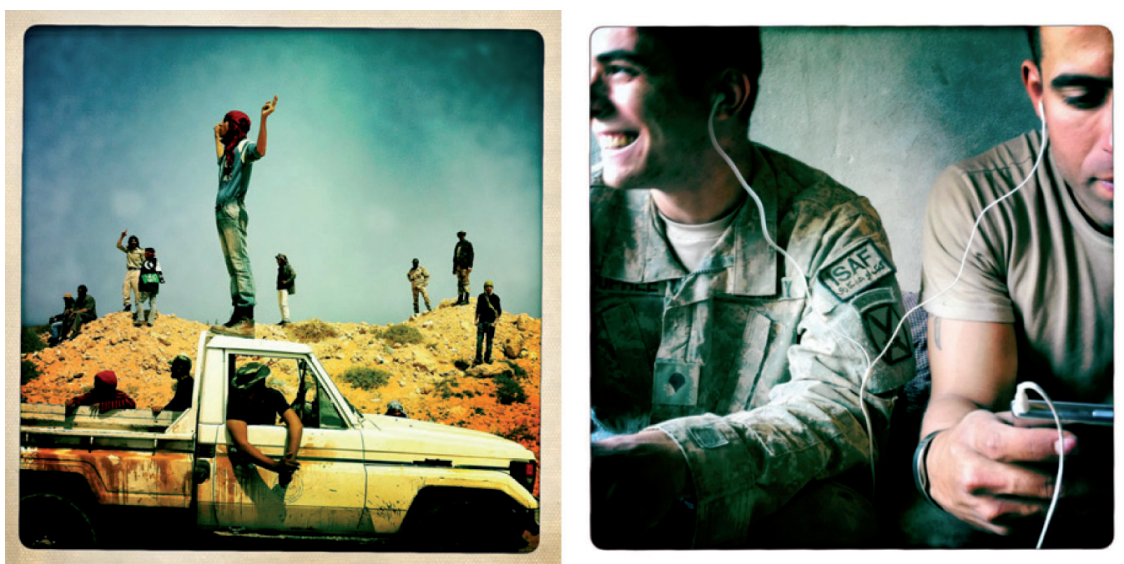

Figure 2 : Reportages iPhonographiques de Ben Lowy et Damon Winter ${ }^{24}$

Depuis les années 1990, le photojournalisme a cru trouver son salut en privilégiant ces symboles canoniques de la reconnaissance institutionnelle: le grand format, le tirage luxueux, l'exposition individuelle, l'affirmation des prérogatives d'auteur, l'entrée dans les grandes collections publiques et privées. Voilà que Damon Winter, en photographiant la guerre à l'Hipstamatic, substitue à ces indicateurs de légitimité ceux, plus valorisants encore, de la déprofessionnalisation volontaire, de la déflation technique, de l'amateurisme candide et de la désuétude esthétique. ${ }^{23}$

Les critiques de Nick Stern et de Matt Buchanan démontrent à quel point une partie du photojournalisme est encore réfractaire à l'idée d'utiliser des applications mobiles comme Instagram ${ }^{25}$ dans la pratique de la profession. Nick Stern oublie pourtant qu'il n'est pas nécessaire que les photographies soient prises par un appareil mobile, ni retouchées par une quelconque application, afin qu'elles soient publiées sur le dispositif numérique. Il est en effet possible de poster une image non retouchée et provenant par exemple d'un reflex professionnel - auquel les photojournalistes sont plus habitués. Instagram n'est donc pas seulement une application de retouches d'images, elle est aussi un dispositif d'exposition de ces mêmes images. Pour le comprendre, il faut à présent expliquer la délimitation de notre corpus d'analyse.

Deux industries médiatiques ont été retenues : Paris Match d'un côté et l'Agence France-Presse de l'autre. Elles sont toutes les deux productrices de contenus photojournalistiques et sont similairement présentes sur Instagram. Leur différence de statut - Paris Match est un magazine d'actualité quand l'AFP est une agence d'information mondiale - nous permet d'observer les possibles

23. Vincent Lavoie, "Guerre et iPhone. Les nouveaux fronts du photojournalisme ", Études photographiques, 29, 2012. URL : http://etudesphotographiques.revues.org/3294

24. Sources : à gauche Ben Lowy en Libye, URL : http://www.benlowy.com/editorial/ilibya/ilibya1-uprising-by-iphone/ ; à droite : Damon Winter en Afghanistan URL : https://lens.blogs.nytimes.com/2010/11/21/finding-the-right-tool-to-tell-a-war-story/?ref=asia

25. Comme ils l'étaient au moment du numérique, voir Anne Beyaert-Geslin, L'image préoccupée, Paris, Lavoisier, 2009. 
points de convergence et de divergence dans la pratique d'un même métier, et ainsi d'élucider le type de relations qu'elles entretiennent différemment avec un dispositif d'exposition numérique comme Instagram.

L'observation s'est effectuée de manière synchronique, en recueillant puis observant les photographies diffusées par ces deux médias sur leurs comptes Instagram durant le mois de septembre 2016. L'analyse privilégie la précision de l'actualité plutôt que l'exhaustivité du corpus. Nous ne nous permettrons donc pas de généraliser les conclusions de l'étude, mais les appréhenderons plutôt avec la distance objective nécessaire à la recherche.

De plus, notre démarche sera multiscalaire. Lorsque nous parlerons de " photographie », ce ne sera pas seulement pour discuter de l'esthétique visuelle de l'image. La photographie s'inscrit dans un support qui a une certaine matérialité, qui est éditorialisée selon certaines règles, avec un format spécifique et un contexte singulier. Le cadre de référence sera aussi important que le visuel exposé, car il conditionne le mode de réception des images. Publier une photographie de presse dans un magazine n'est pas la même chose qu'exposer cette même image sur Instagram. Le dispositif contraint l'image différemment d'un support à l'autre ${ }^{26}$. Deux moments sont donc à distinguer dans l'analyse : l'étude du cadre instituant ${ }^{27}$ dans un premier temps, l'élaboration des effets de sens ${ }^{28}$ dans un second.

\section{Partage: images banales}

La première difficulté à laquelle l'analyse est confrontée est la caractérisation du statut des photographies de presse exposées sur Instagram. Difficile de ne parler que de leur esthétique, tant celle-ci est intrinsèquement déterminée par le double jeu du dispositif d'un côté et de l'héritage photojournalistique de l'autre. De même, par leur publication sur l'application, ces images entrent dans une économie numérique de l'algorithme qui ne correspond plus aux standards traditionnels de la presse et demande par conséquent une nouvelle définition. La proposition de cette enquête préliminaire est généalogique - on pourrait dire philologique ${ }^{29}$. Les images exposées sur Instagram sont des images banales. Non pas «images communes » ou " fades », mais images banales au sens médiéval du terme.

Le banal, du vieux-francique " ban », est en droit féodal ce qui appartient au seigneur et dont l'usage est imposé à ses sujets moyennant redevance ${ }^{30}$. C'est aussi une « chose commune » à la disposition de tous au sein d'une communauté ${ }^{31}$. On comprend que le terme ait pu dériver vers son acceptation actuelle, de quelque chose "d'ordinaire », « commun », « fade » et " courant ». Mais l'étymologie médiévale renvoie plutôt à un espace ou à un objet, qui est partagé par une

26. Voir Annette Beguin-Verbrugge, Images en texte, images du texte. Dispositifs graphiques et communication écrite, Lille, Presses Universitaires du Septentrion, 2006.

27. Voir Yves Jeanneret et Emmanuel Souchier, "L'énonciation éditoriale dans les écrits d'écran ", Communication et langages, 145, 2005, p. 3-15.

28. Voir Pierre Fresnault-Deruelle, L’image manipulée, Paris, collection « Médiathèque », 1983.

29. Mathieu Potte-Bonneville, « Foucault, la vie et la manière », URL : http://www. laviedesidees.fr/Foucault-la-vie-et-la-maniere.html, 7 septembre 2009.

30. CNRTL, 2012.

31. Claude Gauvard, Alain de Libera, Michel Zink (dir.), Dictionnaire du Moyen Âge, Paris, Puf, 2004. 
communauté de personnes contre rétribution - ou non. Le banal inclut donc deux dimensions importantes : l'une économique et l'autre sémiotique.

Si l'on considère son usage pour parler de la photographie de presse numérique, alors cela nous permet d'envisager à la fois la logique de monétisation algorithmique de l'image en tant que produit marchand, et le partage du sens lié à son exposition dans les dispositifs numériques. Une photographie banale, c'est donc une image qui joue de sa matérialité pour circuler, passer le sens et intégrer une logique de monétisation liée au développement des nouveaux dispositifs numériques ${ }^{32}$.

La banalité incarne le statut de la photographie de presse numérique, elle est la combinaison d'un format et d'une matière, un support sémiotique ${ }^{33}$ : labile, protéiforme et soumise à d'éventuelles métamorphoses - qualité d'image, recadrage, retouches. Ainsi soumise au ban des dispositifs numériques, la photographie de presse a subi quelques ajustements dont les conséquences sont économiques, sémiotiques, pratiques et légales. Banalisée, elle est autant sujette aux conflits et aux détournements qu'à son élection symbolique par divers procédés de circulation ${ }^{34}$. Banalisée, elle peut l'être aussi à l'extrême, dans son rapport à la temporalité des médias informatisés comme Instagram.

La banalisation de l'image de presse peut donc se décrire en termes économiques, sémiotiques et spatio-temporels, en questionnant la deixis journalistique à l'heure des dispositifs d'exposition numérique. Qu'est-ce que l'AFP ou Paris Match expriment à travers la banalisation de l'image et qu'est-ce que cela dit de leurs relations aux dispositifs numériques? Quel est le processus mis en œuvre pour y arriver ? Qui détient le pouvoir entre Instagram et Paris Match ? Quelle est la légitimité de l'AFP sur cette même application ? Autant de questions qui nous obligent à déchiffrer les différents « emboîtements » des médiations dans le processus de banalisation de l'image. En se soumettant au dispositif mis en place par Instagram, l'AFP et Paris Match entrent dans un processus économique qui leur échappe et qui sous-tend leurs images. Ils sont dépendants de ce que Dominique Cardon nomme les algorithmes d'autorité et de réputation ${ }^{35}$. Instagram fait autorité dans le domaine du partage des images et il hiérarchise la réputation de ses utilisateurs. Mais si l'AFP et Paris Match se retrouvent à créer des comptes sur l'application, ce n'est que parce qu'Instagram réquisitionne ${ }^{36}$ la pratique de l'exposition d'images sur Internet, obligeant ces deux industries journalistiques à adopter ces nouveaux outils, dans un souci de réputation et de légitimité de leurs pratiques vis-à-vis des utilisateurs d'applications photographiques.

\section{Dominique Cardon, op. cit.}

33. Maria Giulia Dondero et Everardo Reyes-Garcia, «Les supports des images : de la photographie à l'image numérique ", Revue française des sciences de l'information et de la communication [En ligne], 9, 2016, mis en ligne le $1^{\mathrm{er}}$ septembre 2016, consulté le 25 juillet 2017, URL : http://rfsic.revues.org/2124; DOI : $10.4000 /$ rfsic. 2124

34. Adeline Wrona, «Une madone à Fukushima. La condition numérique du portrait de presse », Sur le journalisme, About journalism, Sobre jornalismo, 3(1), 2014. URL : http://surlejournalisme.com/rev

35. Dominique Cardon, op. cit., p. 17-18.

36. La réquisition est « l'ensemble de moyens techniques, idéologiques, formels qui contribuent à pousser toute une société à adopter des outils médiatiques [...] et qui tend à marginaliser ceux qui n'y ont pas recours » dans Yves Jeanneret, Critique de la trivialité. .., op. cit. p. 14. 
L'autorité « réquisitoire » d'Instagram oblige donc l'une des plus grandes agences mondiales à exposer ses photographies sur son application. Certes, en opérant ce déplacement du « fil » professionnel AFP au « fil »d'Instagram, l'enjeu n'est pas purement économique ou autoritaire, il est aussi sémiotique. La photographie de presse est un style avec une tradition, elle a ses propres codes, ses contraintes éthiques et mêmes esthétiques ${ }^{37}$. En décidant de s'installer sur Instagram, l'AFP bouleverse le mode de réception de ses images et gageons que cela bouleverse aussi le mode de captation de ces mêmes images. Il suffit de voir les productions personnelles des photographes de AFP et de les comparer avec ce qu'ils produisent pour elle pour le comprendre. Toute une production habituellement non publiée par l'AFP dans son fil officiel sur le site Image Forum est maintenant disponible sur les comptes Instagram des photographes. Ce faisant, c'est l'image de l'Agence France-Presse et du photojournalisme qui y est pratiqué qui est aussi modifiée.

La banalité est donc un statut qui permet de rallier un certain nombre de questionnements sur la photographie de presse numérique et les liens qui s'établissent entre les dispositifs d'exposition et les industries journalistiques.

\section{DÉPARTAGE : LE PRINCIPE SYNOPTIQUE}

La démarche généalogique nous permet de poser une seconde hypothèse, liée à l'éditorialisation et l'exposition des images sur Instagram. La disposition des photographies rappelle en effet le travail effectué par l'historien de l'art Aby Warburg dans son Atlas Mnémosyne et ses planches photographiques thématiques $^{38}$. Dans notre cas il ne s'agit pas de la diachronie de "la muse Mnémosyne " mais plutôt de la synchronie de la photographie d'actualité et des dispositifs d'exposition numérique.

Il est utile de rappeler que l'objectif de Warburg était de créer une véritable " machine de lecture » afin de repenser l'histoire de l'art à travers un dispositif visuel original et dynamique ${ }^{39}$. Ce qui est intéressant, c'est de voir à quel point les deux projets peuvent trouver des similitudes dans leur approche. Instagram prescrit des tables d'images à ses utilisateurs qui les adoptent en organisant leurs publications comme de véritables planches de style : par motifs, formes, couleurs et thèmes, à la manière d'Aby Warburg dans son Atlas. Paris Match et l'AFP n'y échappent pas non plus ${ }^{40}$, et c'est ici que se joue le départage ${ }^{41}$ de la photographie de presse.

Dans ce départage il y a un travail qui ressemble à l'archivage de l'archéologue. Or dans tout archivage, comme le dit si bien Derrida, il y a violence. Violence du choix, de la suppression d'autres images qui auraient aussi pu être exposées :

37. Vincent Lavoie, Photojournalismes, revoir les canons de l'image de presse, Paris, Éditions Hazan, 2010.

38. Aby Warburg, L'Atlas Mnémosyne, Paris, L'écarquillé, 2012.

39. Georges Didi-Huberman, Atlas ou le gai savoir inquiet. L'œil de l'histoire, 3, Paris, Minuit, 2011.

40. Par l'organisation et l'éditorialisation des effets de sens étudiés plus tard.

41. Dans le sens qui lui a été donné lors de la journée d'étude intitulée « Le partage photographique » au Celsa en octobre 2016 : "Si les pratiques amateurs sont marquées par les logiques de la circulation accélérée des clichés poussée par le discours de la " participation ", ce média semble devoir continuer à suivre les principes du départage des images marquantes ou remarquables, en réaffirmant son rôle de filtre par des gestes éditoriaux en transformation et en réinvention ", URL : http://www.gripic.fr/evenement/partage-photographique 

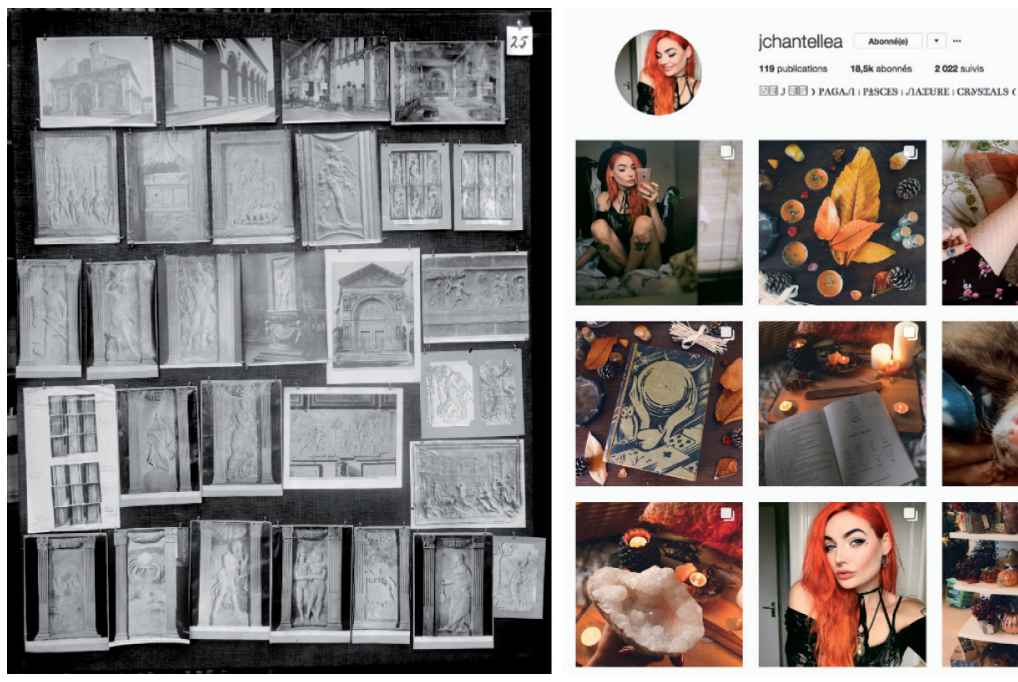

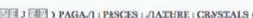
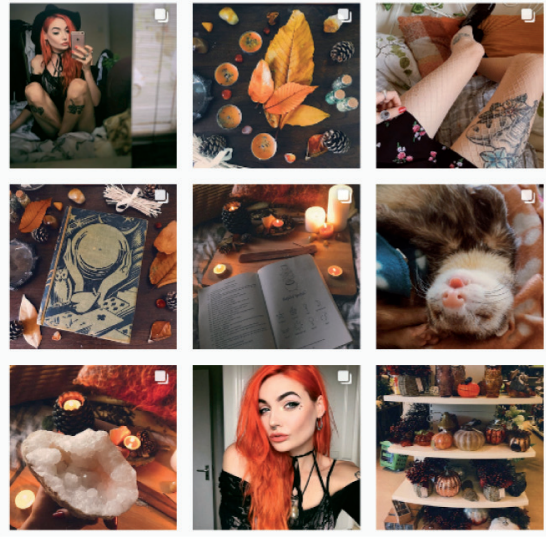

Figure 3 : La généalogie du dispositif d'exposition numérique ${ }^{42}$

L'archive ne traite pas du passé, elle traite de l'avenir. Je sélectionne violemment ce dont je considère qu'il faut que ce soit répété, que ce soit gardé, que ce soit répété dans l'avenir. C'est un geste d'une grande violence. L'archiviste n'est pas quelqu'un qui garde, c'est quelqu'un qui détruit ${ }^{43}$.

Dans ce processus d'archivage des images sur Instagram il y a élection puis désignation : attribution d'une légende, d'une signature, de signes passeurs ${ }^{44}$ désignant le "photographe à suivre ", ou les hashtags à classer. Le fameux " un jour en...» ou «l'instant Paris Match» souvent répétés sur le compte de ce même magazine, ou encore les repost de l'Agence France Presse en sont des exemples. Ces modes de classements organisent des archives photographiques qui fonctionnent selon les codes des tables d'images. C'est pourquoi il est possible de postuler une forme d'archéologie du présent de la sélection, de l'éditorialisation ${ }^{45}$, de la désignation par la légende, de l'archivage par les hashtags, puis de leur exposition sur l'application. D'un point de vue sémiotique, on dirait que le fonctionnement de l'archivage sur Instagram procède par indexation. Annette Beguin-Verbrugge compare les signes indexicaux aux déictiques en linguistique : ils articulent l'espace de l'inscription et l'espace de communication dans laquelle celle-ci prend place ${ }^{46}$. Les images sont indexées par leur message et par la situation de communication

42. Sources : à gauche : planche 25 de L'Atlas Mnémosyne d'Aby Warburg qui assemble et aligne des gravures murales antiques; à droite : capture d'écran du compte Instagram de @jchantellea qui organise ses images selon un code couleur précis et une thématique commune, URL : https://www.instagram.com/jchantellea/?hl=fr

43. Jacques Derrida, Trace et archive, image et art, Paris, Institut National de l'Audiovisuel, 2014, p. 62 44. Jean Davallon et Yves Jeanneret, "La fausse évidence du lien hypertexte », Communication \& langages, 140, 2004, p. 43-54.

45. Les photographies postées par l'AFP sont toutes au format carré, alors qu'on peut les retrouver au format paysage sur leur site de vente d'images.

46. Annette Beguin-Verbrugge, op.cit., p. 56.

communication \& langages - n 194 - Décembre 2017 
dans laquelle elles se trouvent. Le dispositif en tables carrées, symétriques et répétées, conditionne le mode de réception des images de presse sous la forme de l'archivage du présent, du simulacre d'un " passé documenté ».

La bordure extérieure ${ }^{48}$, quant à elle, théâtralise ce qu'elle contient en ce sens qu'elle le donne à contempler. Le seul fait de porter la banalité du quotidien à la « scène » la rend « fascinante » au sens premier du terme, de l' « attrait irrésistible et paralysant exercé par le regard sur une personne ${ }^{49} »$. Une fois encadré et rangé, tout devient signifiant pour le lecteur. Le cadre est comme une injonction à être attentif
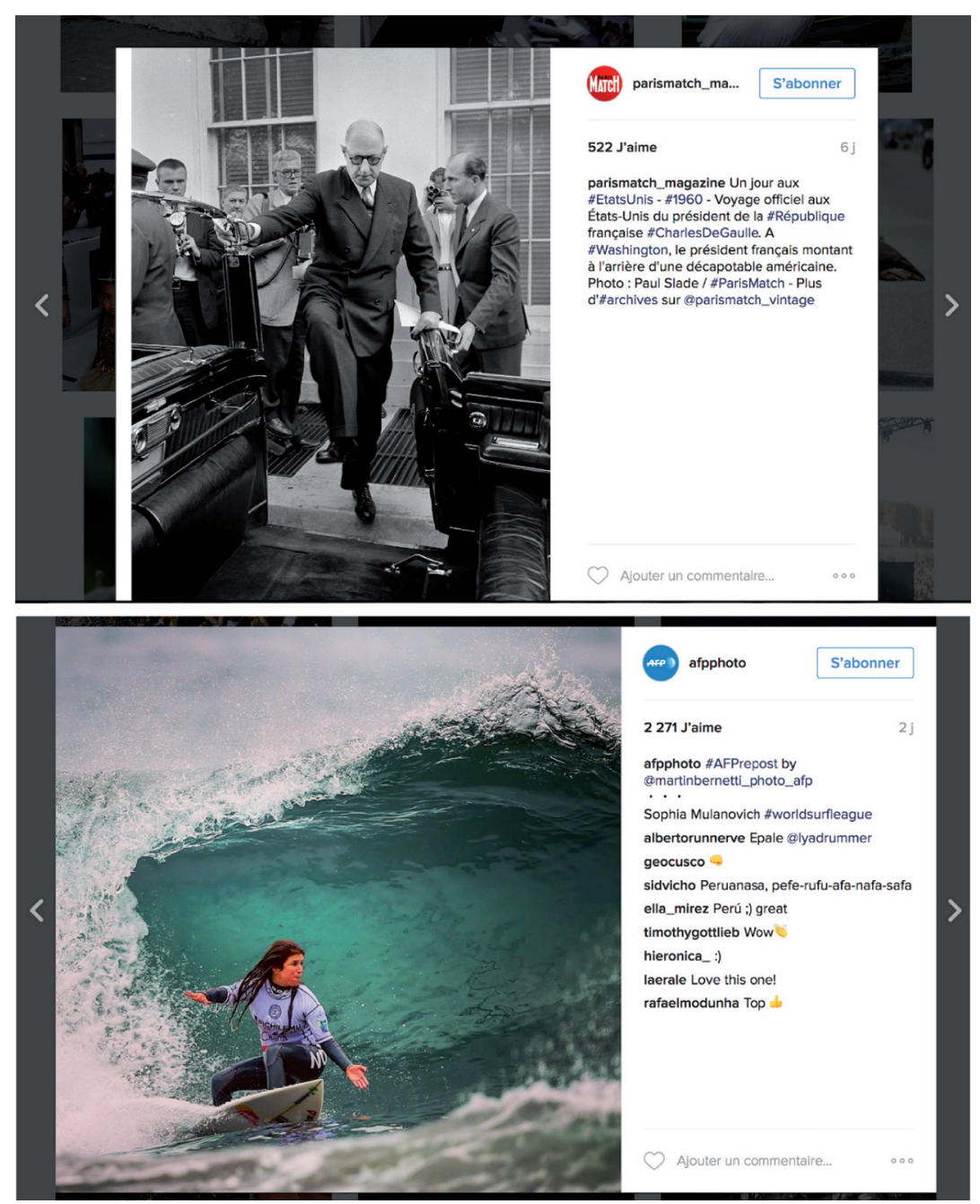

Figure 4 : L'indexation : sélectionner, désigner, archiver, exposer ${ }^{47}$

47. Sources : en haut : «Un jour en... », capture d'écran de l'Instagram de Paris Match, 29 septembre 2016 ; en bas : « \#AFPrepost », capture d'écran de l'Instagram de l'AFP, 29 septembre 2016.

48. Celle qui encadre le texte et l'image, et qui les séparent du site grâce au fond gris en arrière-plan.

49. CNRTL, 2012. 
à ce qui s'y déroule. Ce qui nous amène à présent aux effets de sens produits par le corpus et repérés au cours de l'analyse. Nous en avons délimité quatre.

\section{a. "L'être-ailleurs " des sujets photographiques}

Il concerne le traitement thématique de l'altérité opéré par l'AFP, ou la mondialisation des images sous le joug de la stéréotypie discursive de la photographie de presse $^{50}$ : on puise dans une réserve de stéréotypes pour rendre le sens plus clair.
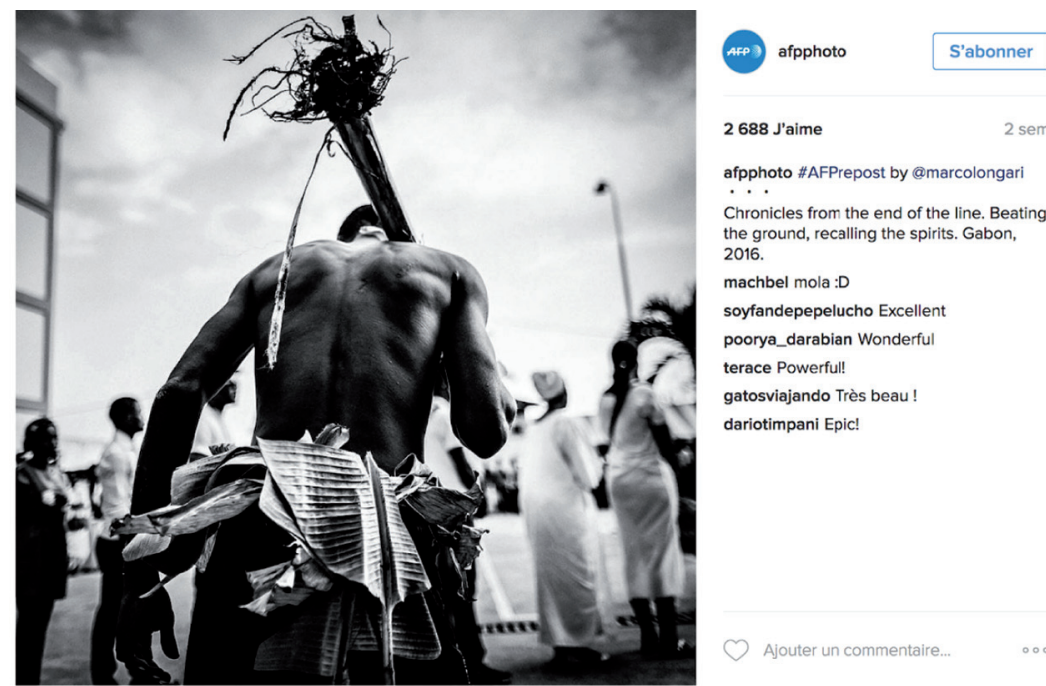

afpphoto \#AFPrepost by @marcolongari

Chronicles from the end of the line. Beating the ground, recalling the spirits. Gabon, 2016.

machbel mola :D

soyfandepepelucho Excellent poorya_darabian Wonderful

terace Powerful!

gatosviajando Très beau !

dariotimpani Epic!

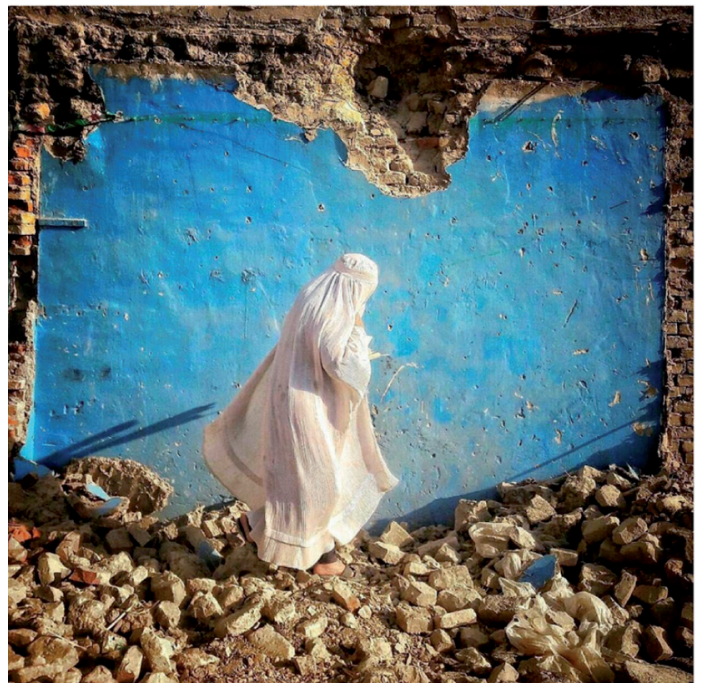

afpphoto \#AFPrepost by @farshadusyan ...

An Afghan burca-clad woman walks through the ruins of a house in Mazari-sharif, Afghanistan.

\#Afghanistan \#nazaresharif sreeranj Excellent shot drmontoya Wow congrats! indieheritage Yo Check Out my new insta video in this account-- @jordnngel vivimur83 $Q$ e tuena foto is shaymaidris Wow ,epic @farshadusyan gentonariel What a picture! Fabulous! miranda_ec @elkinrodriguez andreabuzzichelli STUNNING... aroaamore Bezutiful...and so moving! marekkoerner Like a cloud in the sky.

Figure 5 : « L'être ailleurs ${ }^{51}$ " selon l'AFP

50. Dominique Ducard, "Stéréotypage discursif d'une image de presse », Communication \& langages, 165, 2010, p. 3-14.

51. Sources : captures d'écran de l'Instagram de l'AFP, le 29 septembre 2016. 
Le Gabon est représenté par un danseur folklorique qui «bat le sol »pour « rappeler les esprits », quand l'Afghanistan est incarné par une femme portant une burqa sur les ruines d'une maison a priori bombardée par l'aviation étrangère. Dans les deux cas, on a affaire à la stéréotypie traditionnelle de l'" humanisme exotique du XIX ${ }^{\mathrm{e}}$ siècle » (tel qu'il est défini par Claude Lévi-Strauss dans une note destinée à l'Unesco ${ }^{52}$ ). Ainsi, l'utilisateur abonné au fil de l'AFP est confronté à une réserve de signes bien connus, grâce auxquels le sens est plus clair, sans tension apparente $^{53}$ : le Gabon reste un pays mystique et l'Afghanistan le site d'une guerre religieuse, comme les décrivait déjà « l'humanisme exotique ${ }^{54}$ ».

\section{b. Conformité et totalité : le sens a-tensif}

L'image prend les apparences de la neutralité alors qu'elle n'est que pure mythographie du monde contemporain : prescription et description de "l'humanisme » selon le regard photojournalistique. En publiant cette photographie d'un groupe de danseurs indiens se reliant les mains pour une fête hindoue, Paris Match offre une version claire et apparemment évidente de l'Inde. Elle est conforme aux attentes du public occidental et aux représentations clichées du plus grand pays d'Asie du Sud. La signification est dite " a-tensive » et totale, c'est-à-dire qu'elle limite les interprétations possibles en empêchant tout sens critique de la part du spectateur, qui est obligé de conclure à l'évidence : " c'est bien l'Inde qui est représentée dans cette photographie ». L'Inde est réduite à une totalité significationnelle qui ne semble pas pouvoir être contredite : celle des couleurs
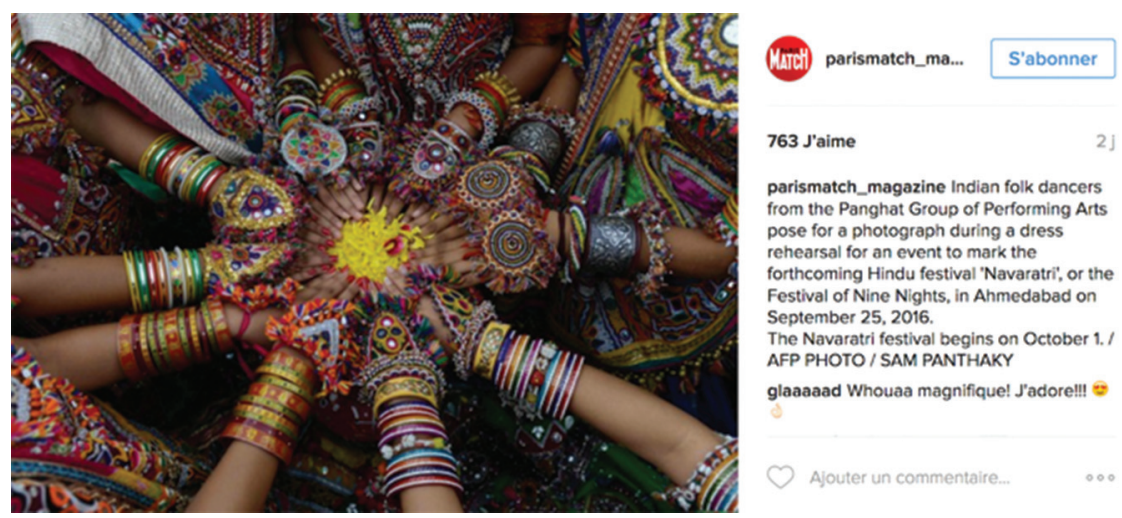

Figure 6 : Le sens a-tensif selon Paris Match $h^{55}$

\section{URL : http://unesdoc.unesco.org/images/0015/001544/154437fb.pdf}

53. Roland Barthes explique à ce propos que l'un des fondements sémiotiques du message photographique de presse réside dans la connotation cognitive, « car la connotation issue du savoir est toujours force rassurante : l'homme aime les signes et il les aime clairs ». Roland Barthes, L'obvie et l'obtus, Paris, Seuil, 1982, p. 22.

54. Voir Tzvetan Todorov, Nous et les autres. La réflexion française sur la diversité humaine, Paris, Seuil, 1989, pp. 353-464.

55. Source : capture d'écran du compte Instagram de Paris Match, 29 septembre 2016. 
vives, des nombreux bracelets traditionnels accrochés aux bras, du sari folklorique et du partage communautaire.

\section{c. L'élection symbolique ${ }^{56}$}

Ces photographies incarnent ce qu'on peut appeler "le sang bleu du photojournalisme » à travers le traitement d'images de guerre, de photographies à l'esthétique "humanitaire » ou de l'occasion sportive. Ces images sont en fait garantes de la disposition synoptique en ce qu'elles la représentent par leur "noblesse " affichée, elles en sont le héraut médiatique et la représentation, en miniature, des productions choisies de Paris Match et l'AFP. La photographie de Mère Teresa à Calcutta montre que Paris Match est un acteur historique du photojournalisme international. Elle renforce la crédibilité du magazine et assoit sa légitimité journalistique, en montrant que le média n'est pas seulement un outil de divertissement, mais aussi un témoin des événements marquants de ces dernières décennies.
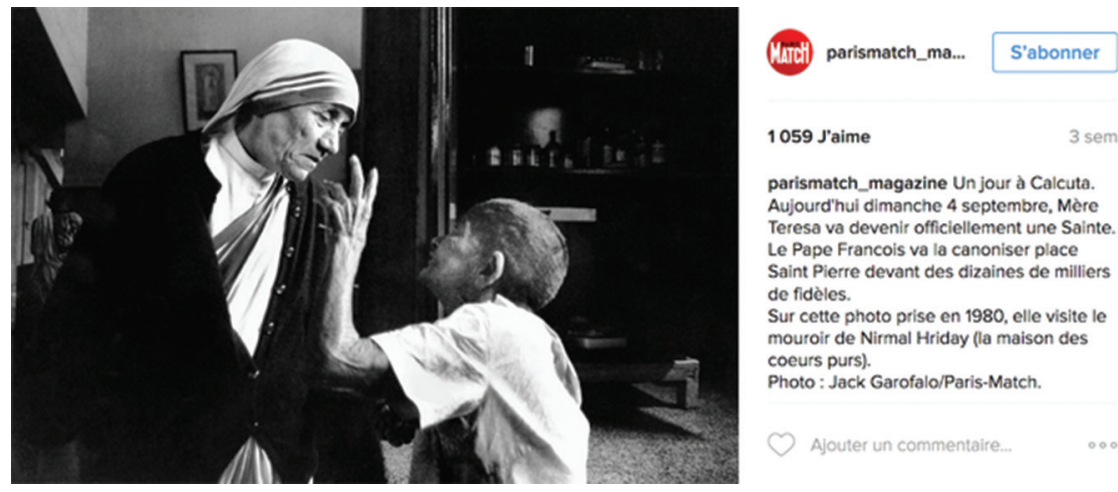

parismatch_magazine Un jour à Calcuta. Aujourd'hui dimanche 4 septembre, Mère Teresa va devenir officiellement une Sainte. Le Pape Francois va la canoniser place Saint Pierre devant des dizaines de milliers de fidèles.

Sur cette photo prise en 1980, elle visite le mouroir de Nirmal Hriday (la maison des

coeurs purs).

Photo : Jack Garofalo/Paris-Match.

Figure 7 : L'élection symbolique selon Paris Match ${ }^{57}$

\section{d. Les effets de surprise}

On sépare ici deux types d'effets de surprise, l'Insolite et l'Occasion.

L'Insolite se définit selon le mode de la "curiosité », comme posture du sujet et comme essence de l'objet ${ }^{58}$. La posture du caribou jouant avec le tuyau d'arrosage et tirant la langue est surprenante, c'est à la fois la rapidité de la prise de vue qui rend la scène insolite, mais aussi l'essence des sujets investis dans l'image : un caribou rencontre un tuyau d'arrosage. La scène interloque.

56. Vincent Lavoie les sépare en deux catégories : le «mérite photojournalistique » et le «protocole pour une photographie compassionnelle » dans Photojournalismes, revoir les canons de l'image de presse, op. cit.

57. Source : capture d'écran de l'Instagram de Paris Match, 29 septembre 2016.

58. Hécate Vergopoulos, Tourisme et curiosités : approche communicationnelle du légendaire dans les guides de voyage imprimés, Thèse de doctorat en Sciences de l'information et de la communication, sous la direction d'Yves Jeanneret et Jacques Pierre, Université d'Avignon, 2010, p. 22-23 


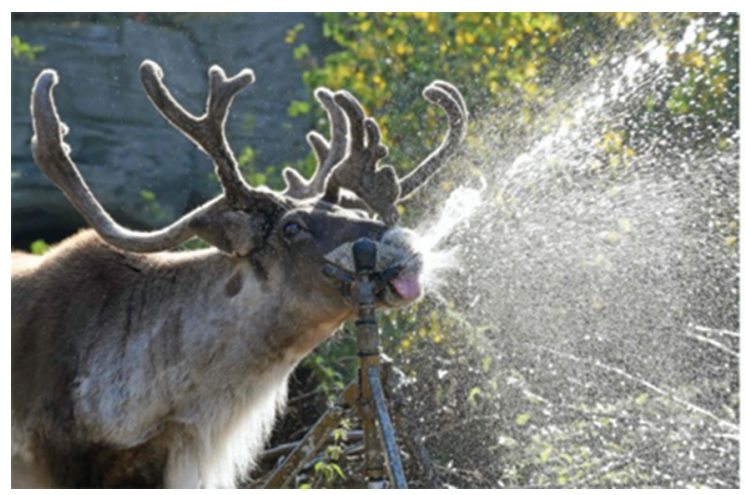

(MATCi) parismatch_ma...

S'abonner

731 J'aime

3 sem

parismatch_magazine Le caribou " Lindsay - se rafraîchit avec un tuyau d'arosage au \#200 de \#Hanovre en Allemagne

"Caribou "Lindsay" refreshes with a water sprinker at the zoo in \#Hanover, northern "Germany, on September 2, 2016. / AFP PHOTO / dpa / Holger Hollemann / Germany OUT

clea.shell aluislocatelli99

Figure 8 : L'Insolite selon Paris Match ${ }^{59}$

L'Occasion c'est la surprise par l'image ou l'exploit photographique de la conjoncture ponctuelle, "l'instant I de l'heure $\mathrm{H}^{60}$ ». L'image de Paris Match ci-dessous repose sur un double exploit : exploit du jeune homme sautant d'une plateforme de 27 mètres, et exploit du photographe réussissant à capturer ce qu'Henri Cartier-Bresson nommait « l'instant décisiff ${ }^{61}$ ». Deux exploits qui se caractérisent par la prise de l'occasion, du synchronisme privilégié du photographe et de son sujet en une image nette et bien composée.

Il est important de noter que ces effets ne se réalisent jamais seuls mais sont toujours en tension, même si l'iconographie photojournalistique tend à l'uniformisation constante de ces points de vue. C'est en cela que l'on parle de principe-synoptique : tout voir, tout décrire, tout savoir ; tels sont les effets de sens produits à la lecture du corpus et du dispositif-Instagram.
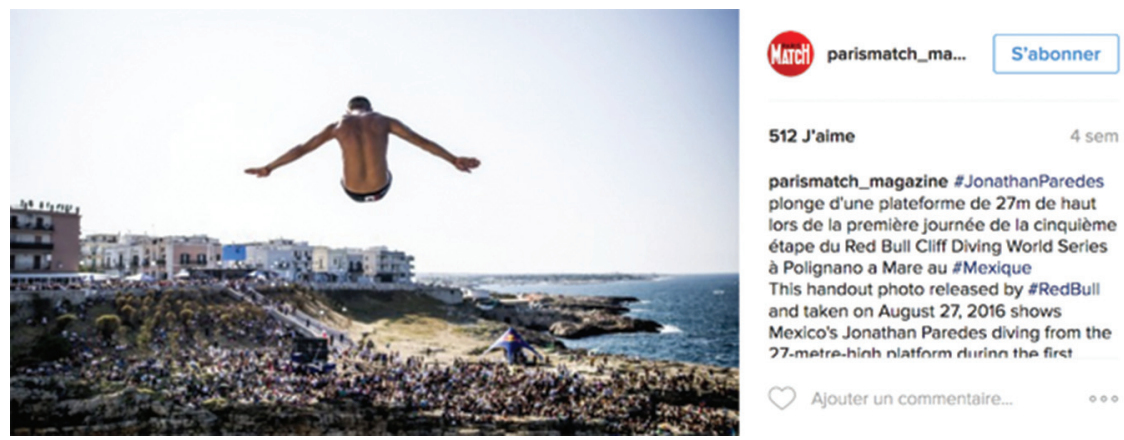

Figure 9 : L'Occasion saisie par Paris Match ${ }^{62}$

59. Source : capture d'écran de l'Instagram de Paris Match, 29 septembre 2016.

60. Vladimir Jankelevitch, Le Je-ne-sais-quoi et le Presque-rien. 1. La manière et l'occasion, Paris, Seuil, 1981, p. 143

61. Henri Cartier-Bresson, Images à la sauvette, Paris, Verve, 1952.

62. Source : capture d'écran de l'Instagram de Paris Match, 29 septembre 2016. 
Ce qui nous intéresse à travers cette hypothèse, c'est l'étendue des productions de l'AFP et de Paris Match, le discours implicite qui est donné sur le monde par le biais de l'image et de son exposition sur les dispositifs numériques. Sans doute cela rejoint-il l'hypothèse sur la banalité de la photographie - son partage -, dans le sens où cette banalité permet et prescrit une réception naturelle des images, alors qu'elles ne sont que des mythographies contemporaines du journalisme. Des mythographies qui sont traitées sous l'angle de l'humanisme photographique et de la construction archéologique du présent, de la construction visuelle de formes de vie mondiales comme la vie animale, la vie insolite ou encore la vie occasionnelle.

\section{Partage et départage de la photographie de Presse sur Instagram}

Le dispositif-Instagram prescrit un mode d'exposition et un mode de réception à travers les concepts et effets de sens que nous avons déployés tout au long de notre démonstration. Mode d'exposition sous le joug de l'archivage du présent et du principe synoptique qui est mis en place par le dispositif ; mode de réception selon les effets de sens déployés par les photographies exposées par Paris Match et l'AFP.

Nous l'avons vu, dans le partage de la banalité il y a une soumission partielle au fonctionnement des algorithmes instaurés par Instagram - et Facebook en amont. Mais d'un autre côté, en départageant leurs photographies, Paris Match et l'AFP s'approprient les codes développés par les utilisateurs d'Instagram. Il n'est donc pas si évident d'opposer d'un côté les industries médiatiques et de l'autre les industries médiatisantes ${ }^{63}$ comme Instagram.

Il faudrait à présent envisager une étude plus large sur la notion de banalité de la photographie de presse numérique. Son application semble en effet poser de nouvelles interrogations quant aux possibilités de sa théorisation communicationnelle. Elle permet en tout cas de cerner une partie des enjeux qui encadrent les relations entre dispositifs numériques d'un côté, et les industries photojournalistiques de l'autre.

\section{Bibliographie}

Agamben Giorgio, Qu'est-ce qu'un dispositif? Paris, Rivages poche, 2014

Barthes Roland, L'obvie et l'obtus, Paris, Seuil, 1982

Beguin-Verbrugge Annette, Images en texte, images du texte. Dispositifs graphiques et communication écrite, Lille, Presses Universitaires du Septentrion, 2006

Beyaert-Geslin Anne, L'image préoccupée, Paris, Lavoisier, 2009

Cardon Dominique, À quoi rêvent les algorithmes. Nos vies à l'heure des big data, Paris, Seuil, 2015

Cartier-Bresson Henri, Images à la sauvette, Paris, Verve, 1952

Davallon Jean et Jeanneret Yves, «La fausse évidence du lien hypertexte », Communication \& langages, 140, 2004

63. Voir à ce propos la distinction opérée par Yves Jeanneret entre les industries médiatiques, médiatisées et médiatisantes : "L’industrie médiatisante se désintéresse des contenus et enjeux de la communication et capte dans ses outils toutes les informations possibles pour les usages ». Yves Jeanneret, Critique de la trivialité..., op. cit., p. 12. 
Certeau De Michel, L'invention du quotidien. 1. arts de faire, Paris, Gallimard

Derrida Jacques, Trace et archive, image et art, Paris, Institut National de l'Audiovisuel, 2014

Didi-Huberman Georges, Atlas ou le gai savoir inquiet. L'œil de l'histoire, 3, Paris, Minuit, 2011

Dondero Maria Giulia et Reyes-Garcia Everardo, "Les supports des images : de la photographie à l'image numérique ", Revue française des sciences de l'information et de la communication, 9, 2016, mis en ligne le $1^{\text {er }}$ septembre 2016, consulté le 25 juillet 2017, URL : http://rfsic.revues.org/2124

Ducard Dominique, "Stéréotypage discursif d'une image de presse », Communication \& langages, 165, 2010

Foucault Michel, Dits et écrits, volume III, Paris, Gallimard, 1994

Fresnault-Deruelle Pierre, L'image manipulée, Paris, collection « Médiathèque », 1983

Gauvard Claude, Libera de Alain, Zink Michel (dir.), Dictionnaire du Moyen Âge, Paris, Puf, 2004

Jankelevitch Vladimir, Le Je-ne-sais-quoi et le Presque-rien. 1. La manière et l'occasion, Paris, Seuil, 1981

Jeanneret Yves et Souchier Emmanuel, «L'énonciation éditoriale dans les écrits d'écran ", Communication \& langages, 145, 2005

Jeanneret Yves, Critique de la trivialité, Le Havre, Éditions Non Standard, 2014

Lavoie Vincent, Photojournalismes, revoir les canons de l'image de presse, Paris, Hazan, 2010

Lavoie Vincent, «Guerre et iPhone. Les nouveaux fronts du photojournalisme », Études photographiques, 29, 2012. URL : http://etudesphotographiques.revues.org/3294

Miles Jason, Instagram power: build your brand and reach more customers with the power of pictures, New York, McGraw-Hill Education, 2013

Potte-Bonneville Mathieu, "Foucault, la vie et la manière », 7 septembre 2009. URL : http://www.laviedesidees.fr/Foucault-la-vie-et-la-maniere.html.

Todorov Tzvetan, Nous et les autres. La réflexion française sur la diversité humaine, Paris, Seuil, 1989

Vergopoulos Hécate, Tourisme et curiosités : approche communicationnelle du légendaire dans les guides de voyage imprimés, thèse de doctorat en Sciences de l'information et de la communication, sous la direction d'Yves Jeanneret et Jacques Pierre, Université d'Avignon, 2010

Warburg Aby, L'Atlas Mnémosyne, Paris, L'écarquillé, 2012

Wrona Adeline, "Une madone à Fukushima. La condition numérique du portrait de presse ", Sur le journalisme, About journalism, Sobre jornalismo, 3(1), 2014. URL : http://surlejournalisme.com/rev

\section{MAXIME FABRE fabre_maxime@live.fr}

